\title{
Plasma waves above the ion cyclotron frequency in the solar wind: a review on observations
}

\author{
C. Briand \\ LESIA, Observatoire de Paris, CNRS, UPMC, Université Paris Diderot; 5 Place Jules Janssen, 92190 Meudon, France
}

Received: 22 December 2008 - Revised: 2 April 2009 - Accepted: 6 April 2009 - Published: 22 April 2009

\begin{abstract}
Since the very beginning of the space venture, discrepancies between hydrodynamic models and observations have raised the attention to the kinetic effects taking place in the interplanetary medium. After discussing some of these constraints, a review on the state of the art knowledge on the waves in the range $f_{c i}<f<f_{p e}\left(f_{c i}-\right.$ about $10^{-1} \mathrm{~Hz}-$ is the ion cyclotron frequency, and $f_{p e}-\mathrm{a}$ few $10 \mathrm{kHz}$ - the electron plasma frequency) observed in the free solar wind, i.e., not magnetically connected to the Earth's bow shock, is addressed.
\end{abstract}

\section{Introduction}

The solar wind is a supersonic, super Alfvénic flow of tenuously magnetized $\left(f_{p e} \gg f_{c e}\right.$, where $f_{p e}$ stands for the electron plasma frequency and $f_{c e}$ for the electron cyclotron frequency), highly ionized and almost collisionless plasma. A first predictive, theoretical model of solar wind was given by Parker (1958) from an hydrodynamic approach (steady state expansion of a thermally driven electron-proton flow out of the hot solar corona). However, this model and the many derived from it were unable to explain the high speed solar wind observed at $1 \mathrm{AU}$ without ad-hoc deposition of momentum and/or energy in the corona. Exospheric/kinetic models have provided better descriptions (Lemaire and Scherer, 1971; Maksimovic et al., 1997; Zouganelis et al., 2004). Moreover, detailed in situ measurements of the shape of the particles distribution function also support kinetic approach. Since the collision length is much larger than the typical interaction length between the waves and particles, the distribution functions are expected to deviate from a Maxwellian shape. Observations indeed reveal that the electron distri-

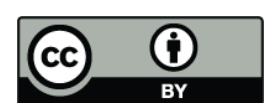

Correspondence to: C. Briand (carine.briand@obspm.fr) bution functions consist of a core (typically below $50 \mathrm{eV}$ ) almost Maxwellian, but also of a halo (representing electrons of higher energy) which strongly departs from a thermal distribution function. In some specific environments (high speed solar wind, far from magnetic sector boundaries), a third component appears: called a "strahl", it represents an enhancement of particles with energies between $100 \mathrm{eV}$ and $1 \mathrm{keV}$ propagating away from the Sun (Pilipp et al., 1987a,b). Moreover, whereas the core of the distributions is rather isotropic, the halo part is highly anisotropic. The high energy particles of the halo present a broad pitch angle while the particles of the strahl are more focused along the magnetic field lines. However, this schematic picture varies considerably according to the solar wind conditions. The proton distribution functions also deviates from a thermal distribution. They show noticeable anisotropies between the temperatures parallel and perpendicular to the magnetic field (Montgomery et al., 1968; Marsch et al., 1981, 1982, and Fig. 1) and present a pronounced field-aligned heat flux or deformations like a bump at high energy.

In situ measurements revealed that the total temperature ${ }^{1}$ anisotropies increase with decreasing heliocentric distances and sometimes exhibit a second proton component that moves along the magnetic field direction with high differential velocities (Marsch et al., 1981, 1982; Marsch, 1991a, and Fig. 1).

The observed deformations of the distribution functions are, however, not as strong as one would expect from the free propagation of the particles. Likely, numerous instabilities develop that limit the scope of the deformations. This means that the plasma somehow reacts to restore stability to the system. Two kinds of back reaction may occur: a

\footnotetext{
${ }^{1}$ It must be stressed that the use of the total temperature (i.e. third moment of the distribution function, integrated over all energy) may contradict the detailed anisotropies when an extended tail exists in one of the parallel or perpendicular component.
}

Published by Copernicus Publications on behalf of the European Geosciences Union and the American Geophysical Union. 

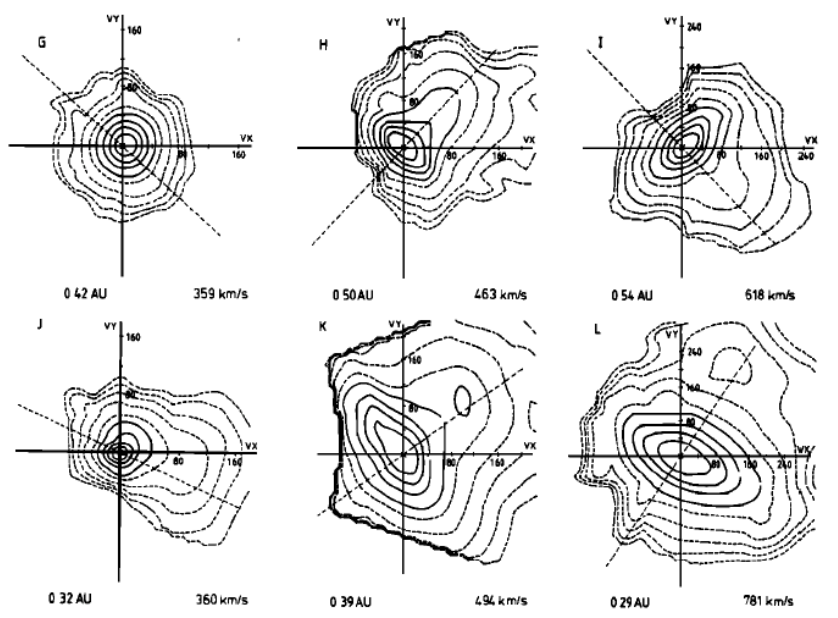

Fig. 1. Helios 2 proton distribution function as measured for low, intermediate and fast solar wind speed (from left to right) and various radial heliocentric distances. Dashed (non contour) line indicates the direction of the magnetic field. From Marsch et al. (1982).

quasilinear relaxation leading to waves with random phases (turbulence) or more complex nonlinear effects which induce coherent structures.

The solar wind energy spectrum shows that the energy is distributed over a broad range of frequencies (Fig. 2). Three zones are usually distinguished following the exponent $\alpha$ of the power law that characterizes each of them: the very low energy regime with $\alpha \approx-1$, the inertial region (roughly $10^{-4} \mathrm{~Hz}<f<f_{c i}$ ) where $\alpha \approx 5 / 3$ and the high frequency domain $\left(f>f_{c i}\right)$ which is thought to be where the energy is finally converted into heat. The evolution of the first part of the diagram (low frequencies) is compatible with the WKB propagation of fluctuations in a spherically expanding solar wind. As the frequency gets closer to about $\approx 10^{-4} \mathrm{~Hz}$ nonlinear coupling between MHD (Alfvén) waves start to be important and allow the cascade of energy through a broader range of frequencies. Finally, above the ion cyclotron frequency, the fluid description of the plasma becomes unsuitable since the phase velocity of the waves is close to the thermal velocity of the particles, thus allowing nonlinear instabilities to develop and energy to transfer between waves and particles. Linking the two regimes (fluid and kinetic) is thus fundamental to follow the energy cascade and to understand the role of the microphysics in the macrostructure of the solar wind (for extended reviews on turbulence in the solar wind, see Marsch, 1991b; Bruno and Carbone, 2005).

The magnetic power spectrum is, however, reliable only up to the ion-cyclotron frequency: above this frequency, the sensitivity of the magnetic field instruments is reduced (as compared to electric field measurements). The high frequency range of the spectrum (corresponding to the dissipative range) is thus better explored through electric field measurements. Intermittent fluctuations of the electric field

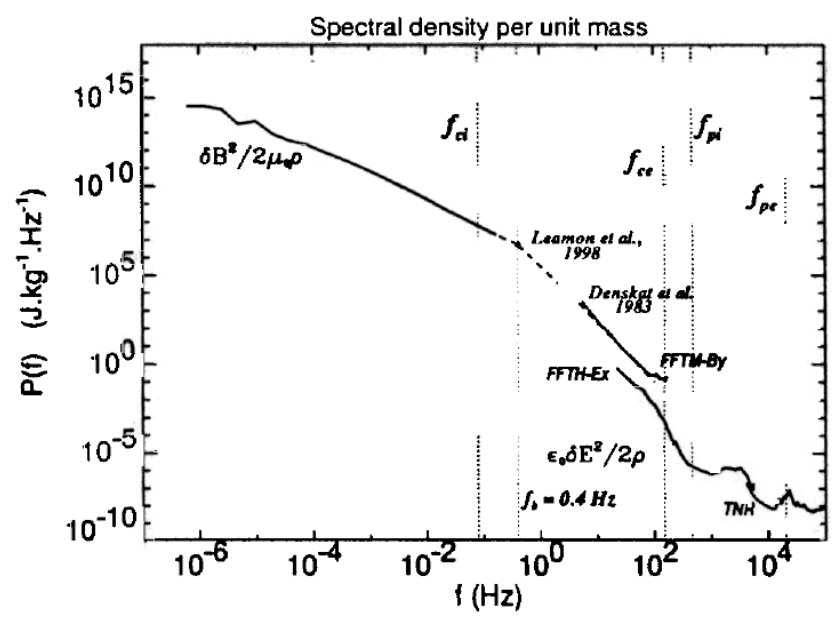

Fig. 2. Magnetic power spectrum observed in the solar wind. From Mangeney et al. (2001).

well above the thermal noise level are indeed observed in the range $f_{c i} \ll f$. Part of these fluctuations are the counterparts of the coherent electrostatic structures observed with waveform analyzers (Lacombe et al., 2002). Linking magnetic and electric power spectrum is far from being a trivial task, since, for example, the contribution of electrostatic waves is not seen in the magnetic spectrum but may represent a large contribution in the higher frequency range.

This paper aims to review the present knowledge on several of the waves in the range $f_{c i}<f<f_{p e}$ from the observational point of view in the free (i.e., not magnetically connected to the Earth bow shock) solar wind. The information obtained from such observations set the fundamental constraints on the most basic physical processes leading to the permanent and large scale structure of the solar wind. This range of frequencies is dominated by electrostatic waves in the higher part (like ion acoustic and Langmuir waves), and includes also electromagnetic waves in the lower part (like whistler-modes and lower hybrid waves). Numerous papers describe these waves in the Earth magnetospheric environment and auroral region but much less exist in the free solar wind (Gurnett and Murdin, 2000).

The paper is organized as follows: after a rapid overview on the different space instruments that have been providing data of the solar wind since the 60's (Sect. 2), a review on the present knowledge on different coherent waves is given. It concerns in particular the Langmuir waves (Sect. 3.1), the ion acoustic waves (Sect. 3.2), the lower hybrid and whistlermode waves (Sect. 3.3) and finally the multipolar isolated electrostatic structures (Sect. 3.4). 
Table 1. List of the space missions that have been providing data on plasma waves in the solar wind. The horizontal line in the table splits up missions with spectrum analyzer (first part) from missions with waveform measurements on board. The peripasis and apoapsis of the orbit of the mission (fifth and sixth column) are given in astronomical units. (*): in December 1983, ISEE-3 was renamed ICE and its main scientific objectives were redefined. $(* *)$ : missions which have provided waveform measurements during their journey in the solar wind while reaching their final destination (Galileo and Cassini) or during excursions outside the Earth magnetosphere (Cluster II).

\begin{tabular}{llllll}
\hline Mission Name & Launch year & End Year & Orbit & Periapsis (AU) & Apoapsis (AU) \\
\hline PIONEER 8 & 1967 & 1996 & Heliocentric near ecliptic plane & 0.992 & 1.088 \\
PIONEER 9 & 1968 & 1983 & Heliocentric near ecliptic plane & 0.754 & 0.99 \\
HELIOS 1 & 1974 & 1981 & heliocentric near ecliptic plane & 0.309 & 0.985 \\
HELIOS 2 & 1976 & 1986 & heliocentric near ecliptic plane & 0.29 & 0.983 \\
ISEE 1-2 & 1977 & 1987 & Highly elliptical geocentric & 1. & 1. \\
ISEE 3 & 1978 & $1983(*)$ & Highly elliptical geocentric & 1. & 1. \\
\hline VOYAGER 1/2 & 1977 & 2007 & Interstellar mission & 1 & - \\
GALILEO $(* *)$ & 1989 & 2003 & Jupiter Orbiter & 1 & 5 \\
ULYSSES & 1990 & 2008 & Solar Polar & 2.3 & 5.4 \\
WIND & 1994 & - & Ecliptic plane & & \\
CASSINI $(* *)$ & 1997 & - & Saturn Orbiter & 1 & 10 \\
CLUSTER II $(* *)$ & 2000 & - & Elliptical geocentric & 1 & 1 \\
STEREO & 2006 & - & Ecliptic plane & 0.9 (sat A) & 1 (sat B) \\
\hline
\end{tabular}

\section{Space instrumentation}

Immediately after the beginning of the space venture during the International Geophysical Year (1957), the exploration of the interplanetary medium started (see Table 1). The PIONEER missions provided the very first in situ measurements of the interplanetary environment albeit still in the Earth vicinity. HELIOS 1 and 2 explored regions down to a distance of 0.3 AU to the Sun (Gurnett and Anderson, 1977) while VOYAGER provided information from the heliosphere well beyond 1AU. This two dimensions view of the interplanetary space was afterwards completed with ULYSSES which probed the unknown regions perpendicular to the ecliptic plane (Wenzel et al., 1992; Marsden et al., 1996).

Low frequency (below the ion gyrofrequency) hydromagnetic instabilities were first studied. The higher frequency domain $\left(f \sim f_{p e}\right)$ attracted more attention when spectrum analyzers and waveform receivers were implemented on board interplanetary missions. Spectrum analyzers give the intensity of the electric field in several frequency channels with narrow bandwidth. The intensity is usually averaged from a fraction of a second (typically $0.5 \mathrm{~s}$ ) to a few seconds ( $36 \mathrm{~s}$ in case of HELIOS for example). Peak intensities are also measured. Such measurements provide continuous measurements over long time intervals(Fig. 3).

However, spectral analyzers lose an important information: the phase of the signal. Waveform analyzers have been implemented since the $90{ }^{2}{ }^{2}$. Contrary to

\footnotetext{
${ }^{2}$ Note that Voyager had a waveform analyzer but the data were mainly used to generate high spectral resolution dynamical spectrum.
}

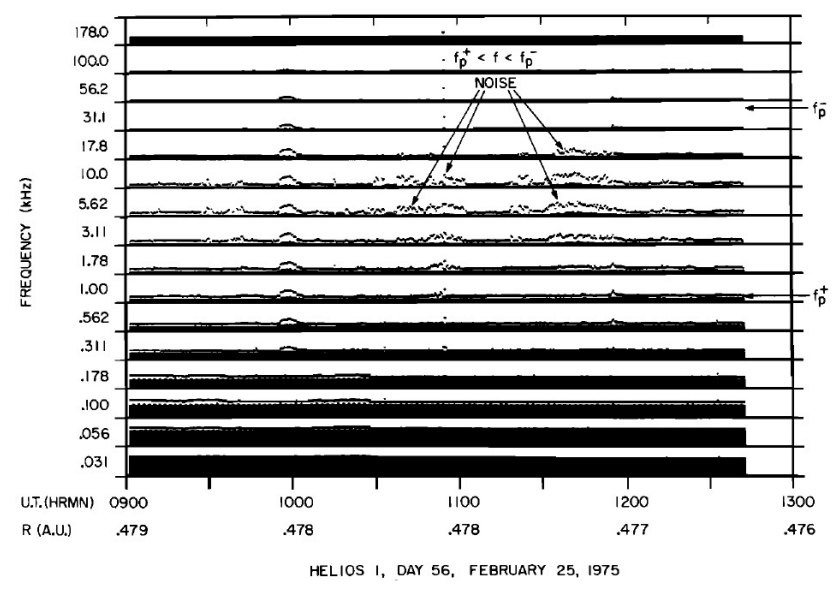

Fig. 3. Example of data obtain by the spectral analyzer on board of HELIOS 1. Black area shows the time average (over $50 \mathrm{~ms}$ ) field strength while the upper curve gives the maximum intensity peak for the same time interval. From Gurnett and Anderson (1977).

the spectrum analyzers, the records of waveforms at high frequency rate are often much shorter. WIND/WAVES (Bougeret et al., 1995), for example, provides waveforms of $15 \mathrm{msec}$ or $230 \mathrm{msec}$ each (see for example Fig. 7) and STEREO/WAVES (Bougeret et al., 2008) provides events $131 \mathrm{msec}$ long. Due to telemetry constraints, one to two hundreds events can be recorded each day. An extremely small fraction of the day (a few seconds at most) is thus covered by the observations. Longer time coverage can, however, be achieved at the expense of the frequency rate (CASSINI/RPWS or CLUSTER/WBD). 


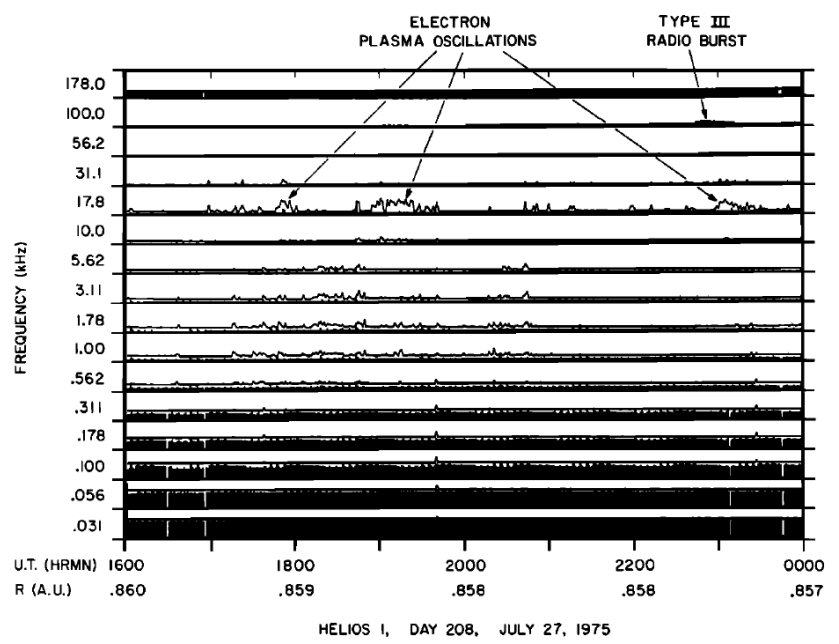

Fig. 4. HELIOS first measurements revealing the presence of Langmuir waves in the solar wind. From Gurnett and Anderson (1977).

\section{In situ measurement of waves in the free solar wind}

\subsection{Langmuir waves}

Langmuir waves are commonly observed in the Earth environment (for example in the vicinity of the bow shock or the foreshock), and they are also a common feature of the solar wind spectrum (Fig. 4). They are observed associated with electron beams generated by flares, beam induced by shocks, or in the presence of magnetic depressions (also called magnetic holes). Langmuir waves are usually thought to result from bump-on-tail instabilities induced by beams propagating in the solar wind. Their dispersion relation is given by:

$\omega^{2}=\omega_{p}^{2}\left(1+3 k^{2} \lambda_{D}^{2}\right)$

$\omega_{p} \propto n_{e}^{1 / 2}$

where $\lambda_{D}$ stands for the Debye length and $n_{e}$ the electron density of the medium.

Since the pioneer work of Ginzburg and Zheleznyakov (1958), Langmuir waves have been intensively studied in relation with Type III radio emission. Indeed, these electromagnetic radio emissions at the plasma frequency, or twice of this frequency, are thought to result from wave coupling implying ion acoustic waves and Langmuir waves. From the observational point of view, numerous works have been devoted to test the validity of this model (Lin et al., 1981, 1986; Gurnett et al., 1993; Thejappa et al., 1993; Cairns, 1995; Thejappa et al., 2003). New evidence of such wave coupling processes have been recently provided from the analysis of waveform data from the STEREO/WAVES experiment (Henri et al., 2009). Since the phase of the waves are available from such measurements, it is possible to test the energy and momentum conservation laws induced by such coupling.

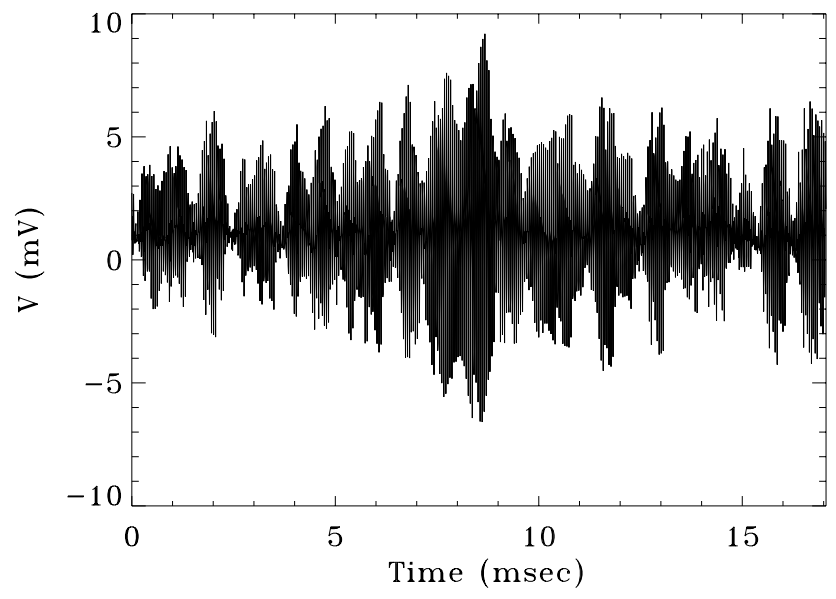

Fig. 5. Langmuir waveform as observed by WIND in the quiet solar wind $(15 / 09 / 2007)$.

As can be expected from the $1 / r^{2}$ variation of the electron density with heliocentric distance $r$, the Langmuir wave peak, observed in the context of Type III bursts, moves towards lower frequencies as the heliocentric distance increases (Fig. 15 of Gurnett and Anderson, 1977). Their amplitude is of a few $\mathrm{mV} / \mathrm{m}$. The development of waveform analyzers has provided more information about these waves. The analysis of waveforms from the WIND/TDS instrument first revealed their coherent nature (Fig. 5), and show that they often appear as highly modulated wavepackets (Kellogg et al., 1999; Mangeney et al., 1999). Recently, Malaspina et al. (2008) proposed a model based on waves trapping in density homogeneities to explain the envelop of Langmuir waves.

Langmuir waves also develop within magnetic holes (Lin et al., 1995; MacDowall et al., 1996). These structures consist of magnetic depressions lasting for a few tens of second up to half an hour. Figure 6 displays such an event as observed by Ulysses. At higher latitudes, the occurrence of Langmuir waves increases in close correlation with the increase of the number of magnetic holes (Fig. 3 of Winterhalter et al., 1994). The amplitude of the electric field decreases with the heliocentric distance to the sun, as in the case of Type III related Langmuir waves (MacDowall et al., 2003). As noted by Lin et al. (1996), the depression of the magnetic field where Langmuir waves occur does not need to be deep (10\% of the ambient magnetic field is enough). More recently, MacDowall et al. (2003) pointed out that Langmuir waves, when not related to particles beams, are mostly encountered in magnetic holes throughout the heliosphere and preferentially in the fast solar wind. The mechanism at the origin of the Langmuir waves within magnetic depression is still unclear. The only model (waves generated by electron beams of thermal energies, formed by adiabatic focusing of the electrons) is indeed unable to also explain the presence of 


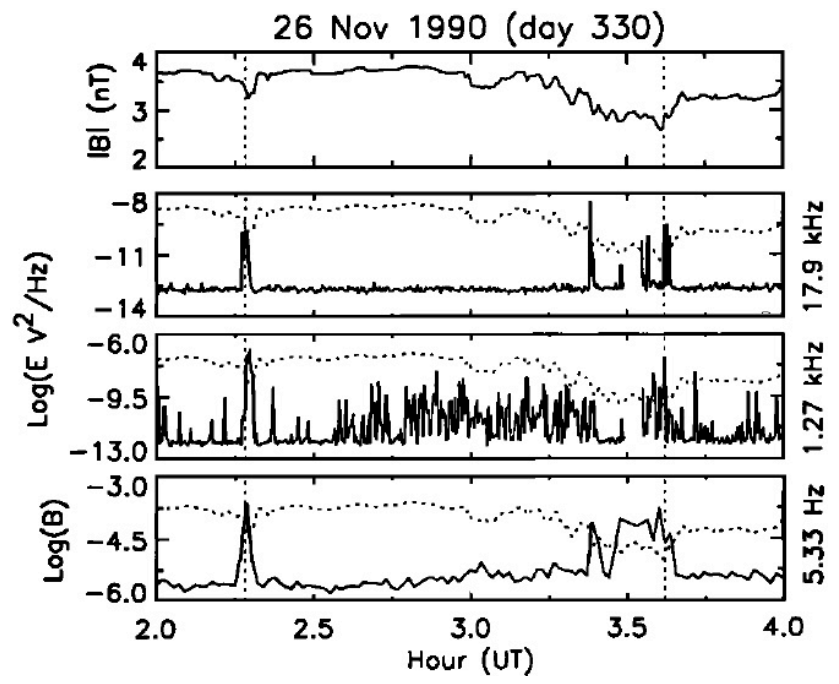

Fig. 6. Time serie of magnetic and electric field measurements from Ulysses. The plasma wave $(17.9 \mathrm{kHz})$ occur in depression in magnetic field (upper panel). The $1.27 \mathrm{kHz}$ electric signal is identified as whistler mode. Note the correlation in time between the occurrence of the two electric signals; From Lin et al. (1995).

other waves often observed simultaneously with the Langmuir waves (and other observational aspects not discussed here).

Finally, Thejappa et al. (2003) have reported Langmuir wave activity not related to either the presence of magnetic holes nor to interplanetary shocks or Type III beams. Such observations encourage statistical studies on Langmuir waves outside obvious periods of electron beam propagation or presence of magnetic holes to complete our knowledge about the formation of Langmuir waves in the solar wind.

\subsection{Ion acoustic waves}

The very first measurements obtained by spectrum analyzers revealed the presence of low frequency turbulence in the quiet solar wind. These emissions, called " $f_{p}^{+}<f<f_{p}^{-}$ noise" by Gurnett and Anderson (1977, where $f^{+}$and $f^{-}$ stands for the local ion and electron plasma frequency respectively), appear as sporadic bursts with a duration from a few minutes to a few days. Figure 3 shows such emissions detected by HELIOS 1 at a distance of $0.47 \mathrm{AU}$ in the quiet solar wind. The " $f_{p}^{+}<f<f_{p}^{-}$noise" is clearly visible between 1.78 and $17.8 \mathrm{kHz}$. The absence of magnetic field signature and the alignment with the interplanetary magnetic field reveal their electrostatic nature. From comparisons with events produced upstream of the Earth bow shock, which show similar characteristics, the waves were finally identified as Ion Acoustic Waves (thereafter IAW), Doppler shifted by advection in the solar wind (Gurnett and Anderson, 1977; Gurnett and Frank, 1978; Kurth et al., 1979).

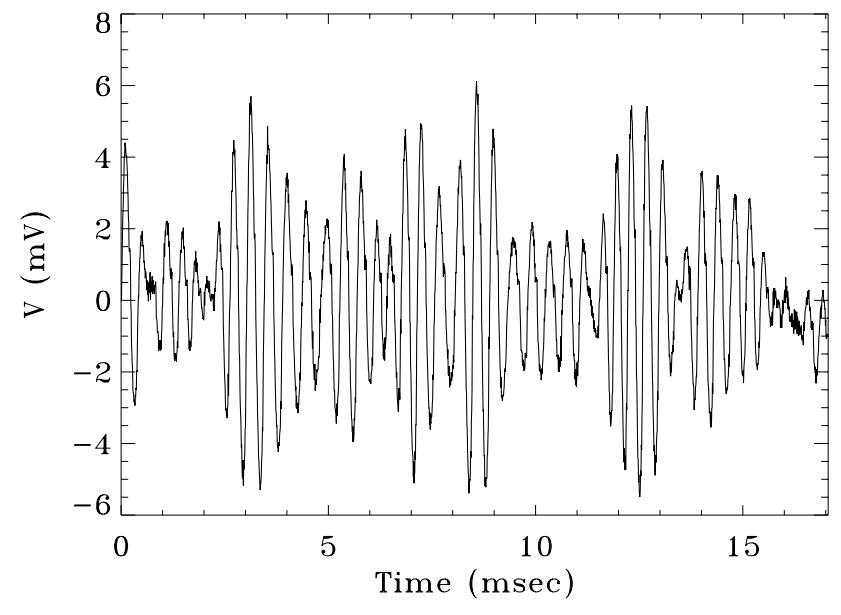

Fig. 7. Example of ion acoustic waveform as observed by the waveform analzer (TDS) on board of WIND.

The intensity of the waves varies between 1 to $10 \mu \mathrm{V} / \mathrm{m}$ in quiet period of solar activity but may reach a few tens of $\mathrm{mV} / \mathrm{m}$ during more active periods. Combined observations from HELIOS I and VOYAGER showed that the intensity of the waves decreases and the frequency peak drifts towards lower frequencies with increasing heliocentric distance (see Fig. 1 of Gurnett et al., 1979). The measurements from ULYSSES also revealed that the IAW activity level depends on the latitude: at low latitude (mostly slow solar wind), the waves present a strong intensity and occur very often, while at higher latitudes (intermediate and fast solar wind) their occurrence and intensity decrease (Lin et al., 2001).

More recently, Mangeney et al. (1999), using the waveform analyzers on board of the WIND spacecraft, gave more details on the characteristics of the IAW. The coherent nature of these waves are obvious from their data (Fig. 7).

A polarization analysis and simultaneous magnetic field measurements confirmed the electrostatic nature of the waves. They estimated the wavelength from the Doppler shift and found $10 \leq \frac{\lambda}{\lambda_{D}} \leq 50$ (where $\lambda_{D}$ stands for the Debye length). They also showed that the wave vector is always directed towards the Earth, independently of the sign of the radial component of the magnetic field. A more surprising result concerns the relationship between the occurrence of these emissions and the proton temperature.

Theoretical arguments, corroborated by numerous simulations, have shown that the ratio of the electron to proton temperature, $T_{e} / T_{p}$, is a fundamental parameter for the stability of the IAW. If $T_{e} / T_{p} \approx 1$, the phase velocity of the waves $\sqrt{\kappa\left(T_{e}+3 T_{p}\right) / m_{p}}$ is very close to the thermal speed of the protons and they are strongly damped by Landau effect. Thus, a necessary condition for the ion acoustic wave to exist is that $T_{e} / T_{p} \gg 1$. Gurnett et al. (1979, their Figs. 7 and 8) indicated that the average peak intensity of the IAW increases with the temperature ratio $T_{e} / T_{p}$. However, according to 


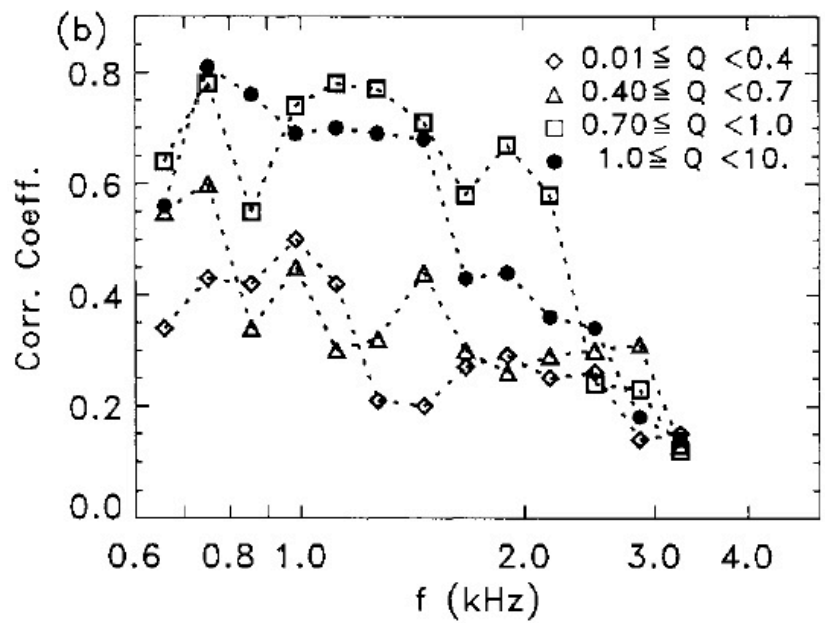

Fig. 8. Correlation of the electric field energy (normalized to temperature energy) in IAW and the ratio $T_{p} / T_{e}$ vs. frequencies for several values of (normalized) electron heat flux. From Lin et al. (2001).

Mangeney et al. (1999) from WIND observations, (i) IAW are measured even when $T_{e} / T_{p} \leq 1$ and (ii) the presence of IA wave packets depends more strongly on $T_{p}$ than on $T_{e} / T_{p}$, with a low value of $T_{p}(\leq 10 \mathrm{eV})$ favoring the waves. In fact, this apparent contradiction between observations results from the fact that in the solar wind, the temperature ratio variations are mainly controlled by the proton temperature, since electrons keep an almost constant temperature of $1-2 \times 10^{5} \mathrm{~K}$.

Lin et al. (2001) analyzed the correlation between ion acoustic waves energy, electron heat flux and electron to proton temperature ratio from ULYSSES data. They concluded that heat flux and temperature ratio are not completely independent parameters (Fig. 8). The correlation with temperature ratio is higher when the electron heat flux is large (when normalized to the energy of the core electron velocity).

Ion Acoustic Waves are also often (but not always) reported in connection to interplanetary shocks (Kurth et al., 1979; Hess et al., 1998; Wilson et al., 2007). The IAW activity is strongly increased at the shock time passage but is also noticeable several hours upstream and downstream of a shock. However, the presence of a shock is not a sufficient condition to generate ion acoustic waves: in several cases, no increase of IAW activity has been noted in spite of the presence of a shock. The occurrence probability of IAW related to interplanetary shocks increases with decreasing latitude but no specific variation with heliocentric distance has been reported. As in the case of the quiet solar wind, temperature of the particles and shape of the distribution functions are the most critical parameters related to the increase of the IAW activity.

Two sources of free energy have been proposed to explain the generation of ion acoustic waves: (i) an electron heat flux
(Forslund, 1970), (ii) an electrostatic double-ion beams instability (Gary, 1978). However, up to now, observations have not been able to disentangle between the two sources. Ion acoustic waves have been observed in regions where the ratio $T_{e} / T_{p}$ would predict strong Landau damping and particle distribution function measurements lack of energy and time resolution to check the stability criterions. The identification of the mechanism(s) at the origin of the ion acoustic waves from direct hydrodynamical conditions of the solar wind is thus still an open question.

\subsection{Lower hybrid and whistler-mode waves}

Lower hybrid waves (also called lower "hybrid" whistlers) and whistler-mode waves are electromagnetic waves propagating parallel to the background magnetic field. At large angle from the magnetic field, the electromagnetic lower hybrid becomes electrostatic. The electromagnetic modes are typical modes found within the electron MHD (where the frequencies are sufficiently high for protons to be considered as fixed). Electromagnetic and electrostatic modes should be distinguished from each other by means of simultaneous measurements of magnetic and electric fields. However, such measurements at low frequencies are hardly available since the sensitivity of the instruments are not always compatible.

Low frequency $\left(f_{c i}<f<f_{c e}\right)$ magnetic signals are a permanent feature of the solar wind, at least close enough to the sun - below 3 AU (Lengyel-Frey et al., 1996). This magnetic activity is enhanced in case of disturbances of the solar wind like near interplanetary shocks (Lin et al., 2003), high-speed streams (Coroniti et al., 1982; Lin et al., 1997; Lengyel-Frey et al., 1996) or magnetic clouds (Moullard et al., 2001). It does not exhibit any specific dependence with latitude (Lengyel-Frey et al., 1996). The spectral density shows a $f^{\alpha}$ power law, with a spectral index $\alpha=-0.65$ in quiet solar wind condition (Beinroth and Neubauer, 1981). From one day average values, Beinroth and Neubauer (1981) showed that the spectral density of the magnetic fluctuations correlate with the proton density and magnetic field strength. Since the correlations disappear in shorter time averages, the spectral density variations reflect the large scale behaviors of these parameters. Lin et al. $(1997,1998)$ also showed that the occurrence of these waves was associated with enhanced heat flux conditions. Moullard et al. (2001) observed whistlermode waves in magnetic clouds. The electric and magnetic waveforms measurements analysis (Fig. 9) reveals that the waves are planar, (right-handed) circularly polarized. Contrary to the results of Coroniti et al. (1982) who found waves propagating at large angle to the magnetic field background, the observations of Moullard et al. (2001) seem to show a propagation aligned with the interplanetary magnetic field. This discrepancy can be explained by an incorrect identification of the waves in the former case. Marsch and Chang (1983) indeed suggested that the waves observed by Coroniti et al. (1982) are more likely lower hybrid waves. 


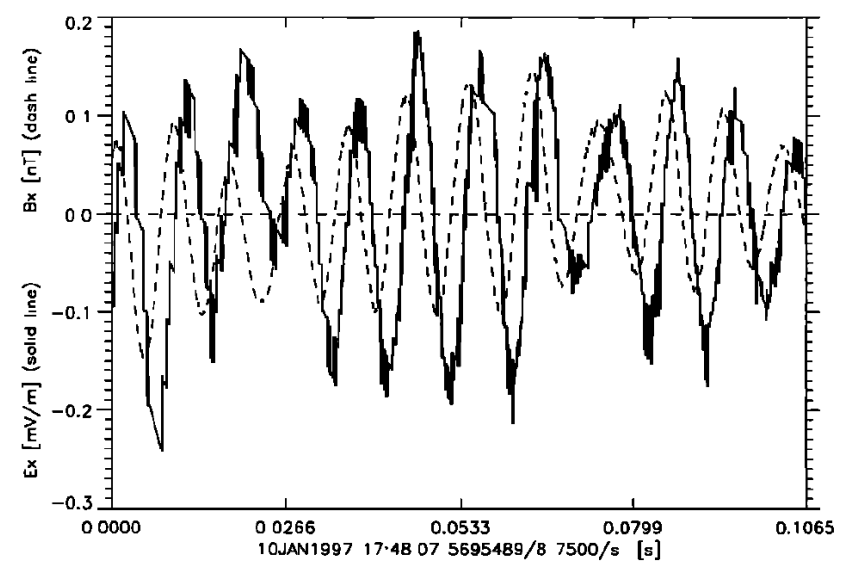

Fig. 9. Example of whistler waveform: Electric field component (solid line) magnetic field component (dashed line). Observed inside a magnetic cloud by the WIND/WAVES(TDS) instrument. From Moullard et al. (2001).

The long-lived background of whistler-mode (or lower hybrid) waves rises the question of the presence of a permanent source of free energy. Loss cone in the electron distribution function, heat flux and electron beams with $T_{\perp} \gg T_{\|}$(and combination of these conditions) are thought to be some possible sources of such free energy. Lengyel-Frey et al. (1996) also suggested that a nonlinear cascade of low-frequency Alfvén waves merging to give higher frequency waves could be at the origin of the background whistler-mode activity. The analysis of the electron distribution function at the time of increased activity of whistler-modes show the presence of loss cone oriented either sunward or anti-sunward (Fig. 7 of Moullard et al., 2001). These observations favor the idea of a local generation of the waves; still a lot of observations and theoretical developments are required to explain the mechanisms leading to the formation of these very low frequency waves.

\subsection{Multipolar electrostatic structures}

We cannot close this review on plasma waves in the solar wind without saying a few words about the bipolar (sometimes tripolar) electrostatic structures (Fig. 10). From the observational point of view they are also referred to as electron, ion or electrostatic solitary waves. In the following, we will prefer the term "isolated bipolar electrostatic structures" (rather than solitary waves) since the mechanism at the origin of these structures is likely to be quite different from the one of solitons. When a net potential drop exists across the structure, they are called double layers.

The first detection of bipolar electrostatic structures and weak double layers was obtained in the auroral region (Temerin et al., 1982). Since then, they are commonly observed in auroral regions (Ergun et al., 1998; Cattell et al.,
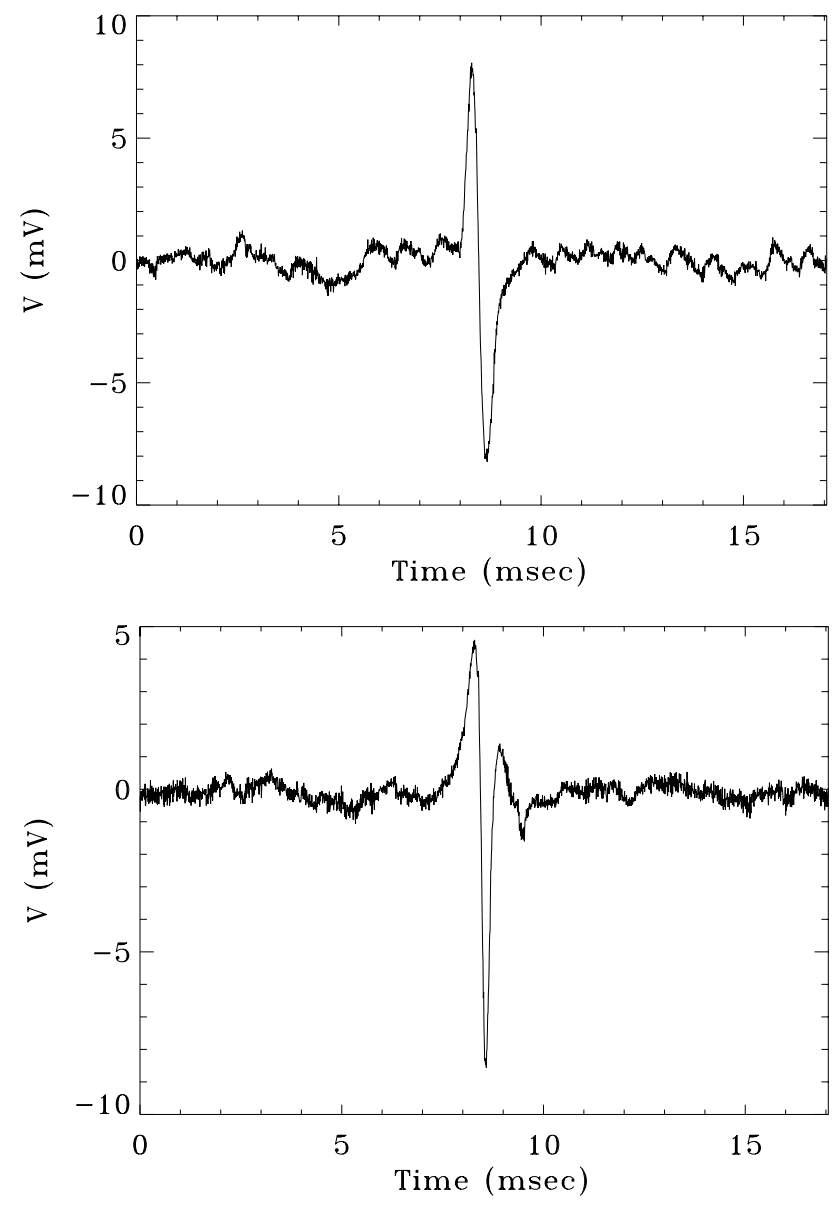

Fig. 10. Examples of isolated electrostatic structures as observed by WIND (15/09/2007): bipolar structures (top panel) and tripolar structures (bottom panel).

2001; Pickett et al., 2004) but also in several Earth magnetic environments (Matsumoto et al., 1994; Franz et al., 1998; Cattell et al., 2003; Pickett et al., 2003) and in the Earth bow shock (Bale et al., 1998; Behlke et al., 2004). Evidence for the presence of isolated bipolar electrostatic structures in the solar wind is more recent: Mangeney et al. (1999) first reported the observation of such structures in the free solar wind and Williams et al. (2006) showed some examples associated to an interplanetary shock. Finally, they were also observed in the vicinity of the magnetosphere of Europa (Kurth et al., 2001) and Saturn (Williams et al., 2006).

From observations with the WIND/WAVES waveform analyzer, Mangeney et al. (1999) deduced that the typical spatial width is about $25 \lambda_{D}$. They also showed that the structures propagate along the magnetic field line at a velocity smaller than the solar wind velocity. Moreover, 29\% of the observed isolated electrostatic structures consists in weak double layers, with a potential jump of a few $\mathrm{mV}^{3}$. The

\footnotetext{
${ }^{3}$ An error of calibration seems to be at the origin of the published tripolar shape. A new calibration of the data was performed and
} 
low potential side is directed towards the Earth and a rough estimate of the total potential difference generated by the succession of such weak double layers from the solar corona to the Earth is consistent with exospheric models (Lacombe et al., 2002). Pickett et al. (2004) performed an extensive statistical analysis of the bipolar and tripolar electrostatic structures observed all along the Cluster II trajectory, which includes auroral zone, polar cusp, polar cap, magnetosheath, plasma sheet and solar wind. They first showed that the electric field amplitude (peak-to-peak) of such structures seem to slightly depend on the magnetic field strength. This could explain why the amplitude of the pulses observed in the solar wind are smaller compared to other magnetopheric environments. They also concluded that the duration of such structures is almost independent of the surrounding, except for the magnetosheath where they are much smaller.

Many questions are still unsolved that require more observations. For examples: what is the origin of these waves (ion/electron phase space holes or electron acoustic solitary waves)? what is the 3-D shape of these structures? 1-D simulations have shown that small structures are easily destroyed to form larger structures, but 2-D simulations demonstrate that, in a weakly magnetized plasma, fast and small amplitude structures are stable while large amplitude waves are unstable (Mottez et al., 1997; Muschietti et al., 1999; Mottez, 2001).

\section{Conclusions}

The waves above the ion cyclotron frequency play a critical role in the microphysics of space plasma. A better understanding of the generation, evolution and decay is required to understand not only the behavior of the plasma at small scale but also to explain the permanent or intermittent behavior of the large scale structures of the solar wind. These waves also play a fundamental role in many physical processes that fall out of the scope of this review (like magnetic reconnection, particles acceleration, Type III radio emissions, etc.). In situ measurements in the region 1-30 solar radii, where basic physical processes are expected to take place are necessary to solve the question of the solar wind heating and acceleration.

As already mentioned, the identification of the waves is often a difficult task for two main reasons. Firstly, contemporaneous measurements of magnetic and electric field are often missing. Secondly, the absence of phase information on the waves limits the studies on the polarization and coupling processes. Waveform analyzers for both electric and magnetic field are thus a requisite to progress any further. In particular, long time coverage events are necessary to study the low frequency range and try to follow the energy cascade from the MHD regime to the kinetic regime. In addition, high energy and temporal resolution of particles distribution

the presence weak double layers was confirmed (Salem, personal communication). functions are necessary to validate possible theories. As can be seen from Table 1 very few instruments will be available in the coming years. Efforts to maintain and develop new instrumentation are necessary to succeed in explaining the solar wind behavior and also Sun-Earth relation.

If more observations are required to impose new constraints on the theories or to validate (or invalidate) some of the already proposed ones, numerical simulations and laboratory experiments are required to understand the, often nonlinear, physics of the medium. The simulations with particles-in-cell, Vlasov-Poisson or hybrid codes have been intensively developed owing to the new capabilities of the computers. In particular, multi-dimensional codes allow now to simulate more realistic situations.

Acknowledgements. The author want to thank C. Salem for providing her a set of newly calibrated WIND/WAVES(TDS) data and for interesting discussions, A. Mangeney and J. L. Bougeret for the careful reading of the paper, and the two referees for their constructive remarks.

Edited by: R. Pottelette

Reviewed by: L. Muschietti and another anonymous Referee

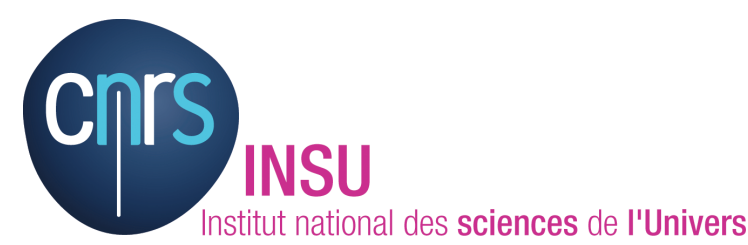

The publication of this article is financed by CNRS-INSU.

\section{References}

Bale, S. D., Kellogg, P. J., Larson, D. E., Lin, R. P., Goetz, K., and Lepping, R. P.: Bipolar electrostatic structures in the shock transition region: Evidence of electron phase space holes, Geophys. Res. Lett., 25, 2929-2932, doi:10.1029/98GL02111, 1998.

Behlke, R., André, M., Bale, S. D., Pickett, J. S., Cattell, C. A., Lucek, E. A., and Balogh, A.: Solitary structures associated with short large-amplitude magnetic structures (SLAMS) upstream of the Earth's quasi-parallel bow shock, Geophys. Res. Lett., 31, 16805, doi:10.1029/2004GL019524, 2004.

Beinroth, H. J. and Neubauer, F. M.: Properties of whistler mode waves between 0.3 and 1.0 AU from HELIOS observations, J. Geophys. Res., 86, 7755-7760, 1981.

Bougeret, J.-L., Kaiser, M. L., Kellogg, P. J., Manning, R., Goetz, K., Monson, S. J., Monge, N., Friel, L., Meetre, C. A., Perche, C., Sitruk, L., and Hoang, S.: Waves: The Radio and Plasma Wave Investigation on the Wind Spacecraft, Space Sci. Rev., 71, 231-263, doi:10.1007/BF00751331, 1995.

Bougeret, J. L., Goetz, K., Kaiser, M. L., Bale, S. D., Kellogg, P. J., Maksimovic, M., Monge, N., Monson, S. J., Astier, P. L., Davy, S., Dekkali, M., Hinze, J. J., Manning, R. E., Aguilar-Rodriguez, E., Bonnin, X., Briand, C., Cairns, I. H., Cattell, C. A., Cecconi, B., Eastwood, J., Ergun, R. E., Fainberg, J., Hoang, S., Huttunen, K. E. J., Krucker, S., Lecacheux, A., MacDowall, R. J., 
Macher, W., Mangeney, A., Meetre, C. A., Moussas, X., Nguyen, Q. N., Oswald, T. H., Pulupa, M., Reiner, M. J., Robinson, P. A., Rucker, H., Salem, C., Santolik, O., Silvis, J. M., Ullrich, R., Zarka, P., and Zouganelis, I.: S/WAVES: The Radio and Plasma Wave Investigation on the STEREO Mission, Space Sci. Rev., 136(1-4), 487-528, doi:10.1007/s11214-007-9298-8, 2008.

Bruno, R. and Carbone, V.: The Solar Wind as a Turbulence Laboratory, Living Reviews in Solar Physics, 2, 1-186, 2005.

Cairns, I. H.: Detectability of Electrostatic Decay Products in ULYSSES and Galileo Observations of Type III Solar Radio Sources, Astrophys. J. Lett., 449, L95-L98, doi:10.1086/ 309621, 1995.

Cattell, C., Crumley, J., Dombeck, J., Lysak, R., Kletzing, C., Peterson, W. K., and Collin, H.: Polar observations of solitary waves at high and low altitudes and comparison to theory, Adv. Space Res., 28, 1631-1641, 2001.

Cattell, C., Neiman, C., Dombeck, J., Crumley, J., Wygant, J., Kletzing, C. A., Peterson, W. K., Mozer, F. S., and Andr, M.: Large amplitude solitary waves in and near the Earths magnetosphere, magnetopause and bow shock: Polar and Cluster observations, Nonlin. Processes Geophys., 10, 13-26, 2003,

http://www.nonlin-processes-geophys.net/10/13/2003/.

Coroniti, F. V., Kennel, C. F., Scarf, F. L., and Smith, E. J.: Whistler mode turbulence in the disturbed solar wind, J. Geophys. Res., 87, 6029-6044, 1982.

Ergun, R. E., Carlson, C. W., McFadden, J. P., Mozer, F. S., Delory, G. T., Peria, W., Chaston, C. C., Temerin, M., Elphic, R., Strangeway, R., Pfaff, R., Cattell, C. A., Klumpar, D., Shelley, E., Peterson, W., Moebius, E., and Kistler, L.: FAST satellite observations of electric field structures in the auroral zone, Geophys. Res. Lett., 25, 2025-2028, doi:10.1029/98GL00635, 1998.

Forslund, D. W.: Instabilities associated with heat conduction in the solar wind and their consequences., J. Geophys. Res., 75, 17-28, 1970.

Franz, J. R., Kintner, P. M., and Pickett, J. S.: POLAR observations of coherent electric field structures, Geophys. Res. Lett., 25, 1277-1280, doi:10.1029/98GL50870, 1998.

Gary, S. P.: Ion-acoustic-like instabilities in the solar wind, J. Geophys. Res., 83, 2504-2510, 1978.

Ginzburg, V. L. and Zheleznyakov, V. V.: On the Possible Mechanisms of Sporadic Solar Radio Emission (Radiation in an Isotropic Plasma), Soviet Astronomy, 2, 653 p., 1958.

Gurnett, D. and Murdin, P.: Solar Wind Plasma Waves, 7280572813, Encyclopedia of Astronomy and Astrophysics, doi:10. 1888/0333750888/2305, 2000.

Gurnett, D. A. and Anderson, R. R.: Plasma wave electric fields in the solar wind - Initial results from HELIOS 1, J. Geophys. Res., 82, 632-650, 1977.

Gurnett, D. A. and Frank, L. A.: Ion acoustic waves in the solar wind, J. Geophys. Res., 83, 58-74, 1978.

Gurnett, D. A., Marsch, E., Pilipp, W., Schwenn, R., and Rosenbauer, H.: Ion acoustic waves and related plasma observations in the solar wind, J. Geophys. Res., 84, 2029-2038, 1979.

Gurnett, D. A., Hospodarsky, G. B., Kurth, W. S., Williams, D. J., and Bolton, S. J.: Fine structure of Langmuir waves produced by a solar electron event, J. Geophys. Res., 98, 5631-5637, 1993.

Henri, P., Briand, C., Mangeney, A., Bale, S. D., Califano, F., Goetz, K., and Kaiser, M.: Evidence for wave coupling in type III emissions, J. Geophys. Res. (Space Physics), 114, 3103, doi:
10.1029/2008JA013738, 2009.

Hess, R. A., MacDowall, R. J., Goldstein, B., Neugebauer, M., and Forsyth, R. J.: Ion acoustic-like waves observed by ULYSSES near interplanetary shock waves in the three-dimensional heliosphere, J. Geophys. Res., 103, 6531, doi:10.1029/97JA03395, 1998.

Kellogg, P. J., Goetz, K., Monson, S. J., and Bale, S. D.: Langmuir waves in a fluctuating solar wind, J. Geophys. Res., 104, 1706917078, doi:10.1029/1999JA900163, 1999.

Kurth, W. S., Gurnett, D. A., and Scarf, F. L.: High-resolution spectrograms of ion acoustic waves in the solar wind, J. Geophys. Res., 84, 3413-3419, 1979.

Kurth, W. S., Gurnett, D. A., Persoon, A. M., Roux, A., Bolton, S. J., and Alexander, C. J.: The plasma wave environment of Europa, Planet. Space Sci., 49, 345-363, 2001.

Lacombe, C., Salem, C., Mangeney, A., Hubert, D., Perche, C., Bougeret, J.-L., Kellogg, P. J., and Bosqued, J.-M.: Evidence for the interplanetary electric potential? WIND observations of electrostatic fluctuations, Ann. Geophys., 20, 609-618, 2002, http://www.ann-geophys.net/20/609/2002/.

Lemaire, J. and Scherer, M.: Kinetic models of the solar wind., J. Geophys. Res., 76, 7479-7490, doi:10.1029/JA076i031p07479, 1971.

Lengyel-Frey, D., Hess, R. A., MacDowall, R. J., Stone, R. G., Lin, N., Balogh, A., and Forsyth, R.: Ulysses observations of whistler waves at interplanetary shocks and in the solar wind, J. Geophys. Res., 101, 27555-27564, doi:10.1029/96JA00548, 1996.

Lin, N., Kellogg, P. J., MacDowall, R. J., Balogh, A., Forsyth, R. J., Phillips, J. L., Buttighoffer, A., and Pick, M.: Observations of plasma waves in magnetic holes, Geophys. Res. Lett., 22, 34173420, doi:10.1029/95GL03266, 1995.

Lin, N., Kellogg, P. J., MacDowall, R. J., Tsurutani, B. T., and Ho, C. M.: Langmuir waves associated with discontinuities in the solar wind: a statistical study., Astron. Astrophys., 316, 425429, 1996.

Lin, N., Kellogg, P. J., MacDowall, R. J., Scime, E. E., Phillips, J. L., Balogh, A., and Forsyth, R. J.: Low frequency plasma waves in the solar wind: from ecliptic plane to the solar polar regions, Adv. Space Res., 19, 877-881, 1997.

Lin, N., Kellogg, P. J., MacDowall, R. J., Scime, E. E., Balogh, A., Forsyth, R. J., McComas, D. J., and Phillips, J. L.: Very low frequency waves in the heliosphere: Ulysses observations, J. Geophys. Res., 103, 12023-12036, doi:10.1029/98JA00764, 1998.

Lin, N., Kellogg, P. J., MacDowall, R. J., and Gary, S. P.: Ion Acoustic Waves in the Heliosphere, Space Sci. Rev., 97, 193196, doi:10.1023/A:1011823505395, 2001.

Lin, N., Kellogg, P. J., MacDowall, R. J., McComas, D. J., and Balogh, A.: VLF wave activity in the solar wind and the photoelectron effect in electric field measurements: Ulysses observations, Geophys. Res. Lett., 30(19), 8029, doi:10.1029/2003GL017244, 2003.

Lin, R. P., Potter, D. W., Gurnett, D. A., and Scarf, F. L.: Energetic electrons and plasma waves associated with a solar type III radio burst, Astrophys. J., 251, 364-373, doi:10.1086/159471, 1981.

Lin, R. P., Levedahl, W. K., Lotko, W., Gurnett, D. A., and Scarf, F. L.: Evidence for nonlinear wave-wave interactions in solar type III radio bursts, Astrophys. J., 308, 954-965, doi:10.1086/ 164563, 1986. 
MacDowall, R. J., Lin, N., Kellogg, P. J., Balogh, A., Forsyth, R. J., and Neugebauer, M.: Langmuir waves in magnetic holes: source mechanism and consequences., in: American Institute of Physics Conference Series, edited by: Winterhalter, D., Gosling, J. T., Habbal, S. R., Kurth, W. S., and Neugebauer, M., vol. 382 of American Institute of Physics Conference Series, 301-304, 1996.

MacDowall, R. J., Lin, N., and McComas, D. J.: Heliospheric Langmuir wave observations from the Ulysses spacecraft, Adv. Space Res., 32, 479-483, doi:10.1016/S0273-1177(03)00331-4, 2003.

Maksimovic, M., Pierrard, V., and Lemaire, J. F.: A kinetic model of the solar wind with Kappa distribution functions in the corona., Astron. \& Astrophys., 324, 725-734, 1997.

Malaspina, D. M., Ergun, R. E., Cairns, I. H., Bougeret, J., Bale, S., Kellogg, P. J., and Kaiser, M. L.: Consequences of the Eigenmode Interpretation of Solar Wind Langmuir Waves, AGU Fall Meeting Abstracts, A1576, 2008.

Mangeney, A., Salem, C., Lacombe, C., Bougeret, J.-L., Perche, C., Manning, R., Kellogg, P. J., Goetz, K., Monson, S. J., and Bosqued, J.-M.: WIND observations of coherent electrostatic waves in the solar wind, Ann. Geophys., 17, 307-320, 1999, http://www.ann-geophys.net/17/307/1999/.

Mangeney, A., Salem, C., Veltri, P., and Cecconi, C.: Intermittency in the Solar Wind Turbulence and the Haar Wavelet Transform, in: Sheffield Space Plasma Meeting: Multipoint Measurements versus Theory, edited by: Warmbein, B., vol. 492 of ESA Special Publication, 53 pp., 2001.

Marsch, E.: Kinetic Physics of the Solar Wind Plasma, 45-133, Physics of the Inner Heliosphere II. Particles, Waves and Turbulence, XI, 352, 152 pp. figs. Springer-Verlag Berlin Heidelberg New York. Also Physics and Chemistry in Space, vol. 21; 2, 45133, 1991a.

Marsch, E.: MHD Turbulence in the Solar Wind, 159-241, Physics of the Inner Heliosphere II. Particles, Waves and Turbulence, XI, 352, 152 pp. figs. Springer-Verlag Berlin Heidelberg New York. Also Physics and Chemistry in Space, vol. 21; 2, 159241, 1991b.

Marsch, E. and Chang, T.: Electromagnetic lower hybrid waves in the solar wind, J. Geophys. Res., 88, 6869-6880, 1983.

Marsch, E., Rosenbauer, H., Schwenn, R., Muehlhaeuser, K.H., and Denskat, K. U.: Pronounced proton core temperature anisotropy, ion differential speed, and simultaneous Alfven wave activity in slow solar wind at 0.3 AU, J. Geophys. Res., 86, 91999203, 1981.

Marsch, E., Schwenn, R., Rosenbauer, H., Muehlhaeuser, K.-H., Pilipp, W., and Neubauer, F. M.: Solar wind protons - Threedimensional velocity distributions and derived plasma parameters measured between 0.3 and 1 AU, J. Geophys. Res., 87, 5272, 1982.

Marsden, R. G., Smith, E. J., Cooper, J. F., and Tranquille, C.: ULYSSES at high heliographic latitudes: an introduction., Astron. \& Astrophys., 316, 279-286, 1996.

Matsumoto, H., Kojima, H., Miyatake, T., Omura, Y., Okada, M., Nagano, I., and Tsutsui, M.: Electrotastic Solitary Waves (ESW) in the magnetotail: BEN wave forms observed by GEOTAIL, Geophys. Res. Lett., 21, 2915-2918, 1994.

Montgomery, M. D., Bame, S. J., and Hundhausen, A. J.: Solar Wind Electrons: Vela 4 Measurements, J. Geophys. Res., 73, 4999-5003, doi:10.1029/JA073i015p04999, 1968.
Mottez, F.: Instabilities and Formation of Coherent Structures, Astrophys. Space Sci., 277, 59-70, doi:10.1023/A:1012224820136, 2001.

Mottez, F., Perraut, S., Roux, A., and Louarn, P.: Coherent structures in the magnetotail triggered by counterstreaming electron beams, J. Geophys. Res., 102, 11399-11408, doi:10.1029/ 97JA00385, 1997.

Moullard, O., Burgess, D., Salem, C., Mangeney, A., Larson, D. E., and Bale, S. D.: Whistler waves, Langmuir waves and single loss cone electron distributions inside a magnetic cloud: Observations, J. Geophys. Res., 106, 8301-8314, doi:10.1029/ 2000JA900144, 2001.

Muschietti, L., Ergun, R. E., Roth, I., and Carlson, C. W.: Phasespace electron holes along magnetic field lines, Geophys. Res. Lett., 26, 1093-1096, doi:10.1029/1999GL900207, 1999.

Parker, E. N.: Suprathermal Particle Generation in the Solar Corona., Astrophys. J., 128, 677 pp., 1958.

Pickett, J., Chen, L., Kahler, S., Santolík, O., Gurnett, D., Tsurutani, B., and Balogh, A.: Isolated electrostatic structures observed throughout the Cluster orbit: relationship to magnetic field strength, Ann. Geophys., 22, 2515-2523, 2004,

http://www.ann-geophys.net/22/2515/2004/.

Pickett, J. S., Menietti, J. D., Gurnett, D. A., Tsurutani, B., Kintner, P. M., Klatt, E., and Balogh, A.: Solitary potential structures observed in the magnetosheath by the Cluster spacecraft, Nonlin. Processes Geophys., 10, 3-11, 2003,

http://www.nonlin-processes-geophys.net/10/3/2003/.

Pilipp, W. G., Muehlhaeuser, K.-H., Miggenrieder, H., Montgomery, M. D., and Rosenbauer, H.: Characteristics of electron velocity distribution functions in the solar wind derived from the HELIOS plasma experiment, J. Geophys. Res., 92, 1075-1092, 1987a.

Pilipp, W. G., Muehlhaeuser, K.-H., Miggenrieder, H., Montgomery, M. D., and Rosenbauer, H.: Unusual electron distribution functions in the solar wind derived from the HELIOS plasma experiment - Double-strahl distributions and distributions with an extremely anisotropic core, J. Geophys. Res., 92, 1093-1101, $1987 b$.

Temerin, M., Cerny, K., Lotko, W., and Mozer, F. S.: Observations of double layers and solitary waves in the auroral plasma, Phys. Rev. Lett., 48, 1175-1179, 1982.

Thejappa, G., Lengyel-Frey, D., Stone, R. G., and Goldstein, M. L.: Evaluation of Emission Mechanisms at omega P E Using ULYSSES Observations of Type III Bursts, Astrophys. J., 416, 831-844, doi:10.1086/173281, 1993.

Thejappa, G., MacDowall, R. J., Scime, E. E., and Littleton, J. E.: Evidence for electrostatic decay in the solar wind at 5.2 AU, J. Geophys. Res. (Space Physics), 108, 1139, doi:10.1029/ 2002JA009290, 2003.

Wenzel, K. P., Marsden, R. G., Page, D. E., and Smith, E. J.: The ULYSSES Mission, Astron. \& Astrophys.s, 92, 207-219, 1992.

Williams, J. D., Chen, L.-J., Kurth, W. S., Gurnett, D. A., and Dougherty, M. K.: Electrostatic solitary structures observed at Saturn, Geophys. Res. Lett., 33, 6103, doi:10.1029/ 2005GL024532, 2006.

Wilson, III, L. B., Cattell, C., Kellogg, P. J., Goetz, K., Kersten, K., Hanson, L., MacGregor, R., and Kasper, J. C.: Waves in Interplanetary Shocks: A Wind/WAVES Study, Physical Review Letters, 99, 041101, doi:10.1103/PhysRevLett.99.041101, 2007. 
Winterhalter, D., Neugebauer, M., Goldstein, B. E., Smith, E. J., Bame, S. J., and Balogh, A.: ULYSSES field and plasma observations of magnetic holes in the solar wind and their relation to mirror-mode structures, J. Geophys. Res., 99, 23371-23381, 1994.
Zouganelis, I., Maksimovic, M., Meyer-Vernet, N., Lamy, H., and Issautier, K.: A Transonic Collisionless Model of the Solar Wind, Astrophys. J., 606, 542-554, doi:10.1086/382866, 2004. 